\title{
Somatisierungsstörungen \\ und psychische Komorbidität \\ Diagnose und Therapieoptionen am Beispiel türkischer Patienten in Deutschland
}

\author{
Vahndet Gül1', Semra Kolb², Havva Saglam³ \\ 1 Facharzt für Psychiatrie, Mühlacker \\ 2 Fachärztin für Psychiatrie und Neurologie, Stuttgart \\ ${ }^{3}$ Fachärztin für Psychiatrie und Neurologie, Frankfurt am Main
}

Die Somatisierung wurde lange Zeit als eine chronisch fluktuierende Störung ohne entsprechende objektivierbare Befunde angesehen. Die Symptome werden dabei als eine körperliche Erkrankung wahrgenommen, ohne dass pathologische Befunde erhoben werden können. Die Folge ist, dass viele unnötige und oft intensive diagnostische und therapeutische Maßnahmen unternommen werden, jedoch ohne dass sich der gewünschte Erfolg einstellt $(13,33)$.

\section{Vorraussetzungen für die Entwicklung „kulturkompatibler“ Interventions- möglichkeiten}

Im westlichen Krankheitsverständnis werden körperlichen Symptomen organische Ursachen zugeordnet. Im Gegensatz dazu besteht in der östlichen Kultur eine deutliche Tendenz, organische Beschwerden als einen Ausdruck einer seelischen Störungen wahrzunehmen (5).

Neuere Studien betonen den Stellenwert von Ethnizität und Kultur sowohl als potenzielle Auslöser einer Somatisierungsstörung als auch in der erfolgreichen Behandlung derselben (35). Der sozioökonomische Status ist ebenfalls von Bedeutung. Bei Patienten mit Somatisierungsstörungen liegt eine Vielzahl an ursächlichen Faktoren vor wie z.B. ein geringer Bildungsstatus, ärmliche häusliche Verhältnisse, ein geringes Einkommen, Arbeitslosigkeit und chronisch latente soziale Konflikte.

Die Fähigkeit zur kulturellen Sensibilität entsteht aus dem Verständnis des Arztes für seinen Patienten durch Kenntnis seines Glaubens, seiner Art der Gesundheitsvorsorge, seiner Stereotypien und seiner sozialen Umgebung. Das Wissen um die Einwanderungsmotive (z.B. politische oder wirtschaftliche Konflikte) kann diese Sensibilität noch erhöhen (27).

Unter Berücksichtigung all dieser Fakten ergeben sich für alle Beteiligten des Gesundheitssystems neue Möglichkeiten für die Entwick-
In der Diagnose und Therapie der Somatisierungsstörung wurden bereits deutliche Fortschritte erzielt. Ethnische und kulturelle Aspekte bringen jedoch eine andere Dimension in die Psychopathologie dieses Krankheitsbildes. Insbesondere die Komorbidität spielt in der Pathologie, beim klinischen Erscheinungsbild, der Behandlung und Prognose eine große Rolle. Es wird vermutet, dass bei einer therapieresistenten Somatisierungsstörung zumindest eine psychiatrische Komorbidität vorliegt. Für den Fall, dass eine Komorbidität vorliegt, wird empfohlen, zunächst die Komorbidität zu behandeln, damit es auch zu einer Heilung bzw. Linderung der Somatisierungsstörung kommen kann. Obwohl bereits viele Publikationen über Somatisierung und Ethnizität vorliegen, gibt es bedauerlicherweise nur wenige Publikationen über Somatisierungsstörung und Komorbidität, insbesondere über psychiatrische Komorbidität bei Migranten, hier speziell bei türkischen Patienten. Diese Studie beabsichtigt die, psychiatrische Komorbidität zu erfassen und die Aufmerksamkeit auf die sozialen und kulturellen Besonderheiten bei türkischen Patienten mit einer Somatisierungsstörung in Deutschland zu richten.

lung „kulturkompatibler“ Interventionen.

Für Patienten mit einer Somatisierungsstörung wird postuliert, dass der Prozess der Umsetzung eines unspezifischen Stresses in ein physiologisches Erregungsniveau oder in eine physische und psychische Symptomatik gestört ist (20). So kann eine Somatisierungsstörung mit einer Angststörung, einer Depression oder einer Funktionsstörung vergesellschaftet sein $(13,28)$ und es besteht für den Patienten mit einer Somatisierungsstörung die Gefahr von dem Therapeuten als Simulanten deklariert zu werden (5). Somatisierung tritt bei ethnischen Minderheiten häufiger auf im Vergleich zur Gesamtbevölkerung $(5,12,38)$.

\section{Somatisierung und psychische Komorbidität}

Die türkische Gemeinschaft - mittlerweile in der vierten Generation - bildet die größte eth-

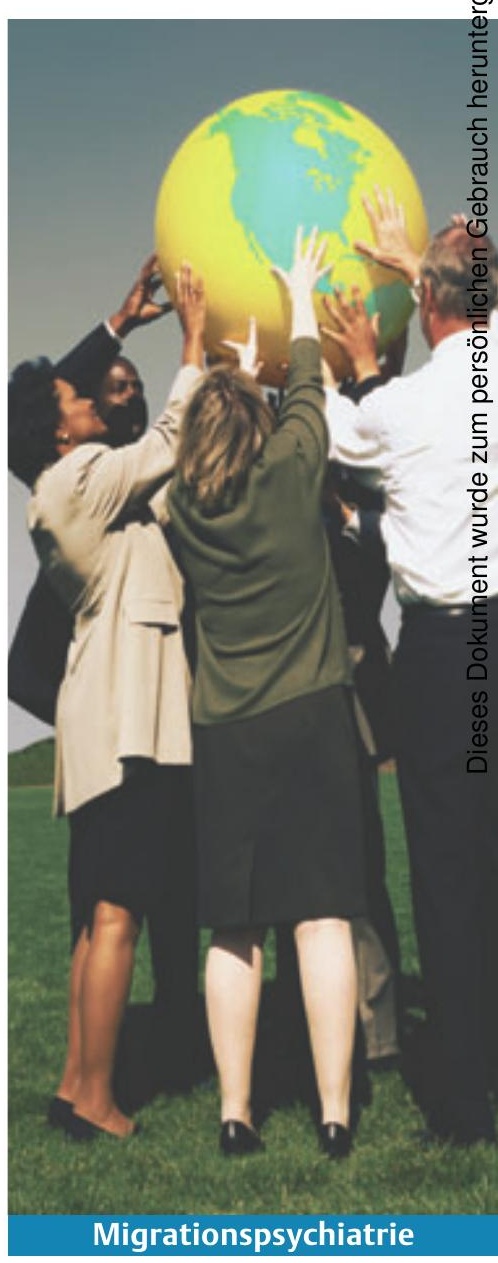


nische Minderheit in Deutschland. In dieser heterogenen Gruppe gibt es gravierende Unterschiede bezüglich Alter, Geschlecht, religiöser Gesinnung, sozioökonomischem Status, Ausbildung und Aufenthaltsdauer in Deutschland. Die Unfähigkeit eines Patienten, seine seelische Qual zu verbalisieren führt in niedrigeren sozialen Schichten tendenziell dazu, die Störung somatisch zu präsentieren. Auch gab es viele Diskussionen über die vielzitierte Tendenz von türkischen Mitmenschen, psychische Symptome als somatische zu präsentieren $(1,19,35,36)$. Durch die vermehrte Zuwanderung kam es in den letzten 15 Jahren zu einem Wandel der bisherigen Strukturen; die Unterstützung durch die Familie und die Gemeinschaft bei der Bewältigung von Schwierigkeiten stand nicht mehr zur Verfügung. Mangelnde Sprachkenntnisse und die Unwissenheit über das deutsche Gesundheits- und Sozialsystem waren zudem hohe Barrieren, insbesondere für die neuen türkischen Migranten (22, 23, 26).

Einige psychische Störungen können bei ethnischen Minderheiten durch den Glauben und den kulturellen Rahmen weiterhin verstärkt werden. Der Einfluss von religiösem Glauben und ethnischer Zugehörigkeit kann in den verschiedenen Kulturen variieren. Es kommt selten vor, dass sich ihre Mitglieder öffentlich über ihr Befinden beklagen. Die Gründe hierfür liegen einerseits an der Sprachbarriere, andererseits auch an der Angst vor Stigmatisierung. Dies ist besonders innerhalb der türkischen Gemeinschaft der Fall (1, 23, 31, 36).

Ungelinderte somatischen Beschwerden wiederum führen zu einer angespannten Beziehung zwischen Patient und Arzt. Oft fehlt dem Arzt das nötige Verständnis für die Krankheit seines türkischen Patienten in Zusammenhang mit seiner kulturell-religiösen Überzeugung sowie seines Aberglaubens (1, 2, 7, 11, 38). Mögliche Folgen sind ein sogenanntes Doctorhopping mit entsprechend unterschiedlichen Diagnosestellungen und Therapien. Der Patient ist

Tab. 1 Somatisierungsstörung und psychiatrische Komorbidität bei türkischen Patienten in Deutschland

Nebendiagnose nach ICD-10
F32 Depressive Störungen
F34.1 Dysthymia
F41 Angststörungen
F43.2 Anpassungsstörungen
F51.0 nichtorganische Insomnie
Alle anderen psychischen Störungen
Gesamt
Geschlecht

5-Jahres-Prävalenz

$27,0 \%(n=62)$

$7,0 \%(n=16)$

$9,5 \%(n=22)$

$4,3 \%(n=9)$

$1,8 \%(n=4)$

$5,2 \%(n=12)$

$54,8 \%(n=125)$

$58,2 \%$ männlich $(n=134)$

$41,7 \%$ weiblich $(n=96)$ zunehmend frustriert und belastet so zusätzlich die Ressourcen des Gesundheitssystems.

Die Rate der psychiatrischen Komorbidität, insbesondere die der Depression und Angststörung, steigt mit der Anzahl der körperlichen Beschwerden (3, 8, 10, 13, 28). Einige Untersuchungen zeigen einen linearen Zusammenhang zwischen den unerklärlichen somatischen Symptomen und dem Auftreten von depressiven Störungen oder Angststörungen (18, 29, 32, 34, 37).

In einer Studie über Patienten mit einer Somatisierungsstörung, die in Praxen von Allgemeinärzten, Hausärzten und z.T. Orthopäden behandelt wurden, zeigte sich bei $54 \%$ der Patienten zusätzliche eine Depression, bei 33,6\% eine generalisierte Angststörung und bei 31,1\% eine Phobie $(4,6)$. Es ist von elementarer Bedeutung verstärkt nach diesen Komorbiditäten zu suchen, da sie auf eine psychopharmakologische Therapie ansprechen und dadurch auch zu einer günstigen Prognose der Somatisierungsstörung führen können $(7,24)$. Es gibt leider noch sehr wenige Publikationen über Somatisierungsstörung in Zusammenhang mit Komorbidität, insbesondere über psychiatrische Komorbidität bei türkischen Patienten.

Tabelle 1 gibt eine Übersicht der Komorbiditätsraten, die bei 230 untersuchten türkischen Patienten mit Somatisierungsstörungen evaluiert wurden. Etwa jeder dritte türkische Patient mit einer Somatisierungsstörung zeigte zusätzlich eine depressive Episode. Im Durchschnitt lag bei ca. $54 \%$ eine Komorbidität vor.

\section{Therapieziele}

Kognititve Verhaltenstherapie in der Muttersprache

Die kognitive Verhaltenstherapie (KVT) in der Muttersprache ist unter Berücksichtigung der kulturellen und religiösen Aspekte bei der Behandlung die Therapie der ersten Wahl. Ihr Ziel ist nicht, die Symptome vollständig zum Abklingen zu bringen, sondern über gezielte Lösungsmehoden zu lernen, diese im Alltag besser zu ertragen nach dem Motto „Rather care, than cure" $(9,14,21,30)$.

Ziel der kognitiven Verhaltenstherapie ist es,

- den somatischen Stress zu reduzieren,

- das maladaptive Verhalten der Kranken, welches von Religion und Kultur beeinflusst wurde, zu korrigieren,

- einer schweren Chronifizierung und einer sozialen Funktionsstörung im Alltag vorzubeugen.

Die KVT befähigt die Patienten,

- den Zusammenhang zwischen Stress und der Zunahme der körperlichen Symptome zu erkennen, 
- eigene Widerstände, die aus persönlichen ethno-kulturellen und religiösen Überzeugungen herrühren zu erkennen,

- mithilfe einer gezielten Anwendung von Stress-Management-Techniken und Entspannungsverfahren mit Stress im Alltag lernen umzugehen.

\section{Medikamentöse Therapie}

Besteht eine psychiatrische Komorbidität (z.B. Depression oder Angststörung), empfiehlt sich eine sofortige entsprechende psychopharmakologische Behandlung, da sie auf eine medikamentöse Therapie ansprechen, und auch zu einer günstigen Prognose der Somatisierungsstörung führen $(7,24)$. Viele Studien raten in der Behandlung von bestimmten funktionellen somatischen Beschwerden (z. B. irritablem Reizdarmsyndrom, Chronic-Fatigue-Syndrom, Fibromyalgia) zu dem Einsatz von Antidepressiva, wobei es hierbei keine Überlegenheit der Serotonin-Wiederaufnahmehemmer (SSRI) gegenüber den trizyklischen Antidepressiva (TCA) gibt $(16,25)$. Fehlt eine psychiatrische Komorbidität wie z. B. eine Depression oder Angststörung, ist die Indikation für eine antidepressive Medikation nicht eindeutig. Hier wird in der Regel ein niedrig dosiertes Antidepressivum verabreicht und langsam aufdosiert (16).

\section{Literatur}

1 Ayan SJ, Calliess IT. Multikulti der Seelennöte. Gehirn \& Geist 2004; 2: 16-19

2 Barsky AJ, Ahern DK. JAMA 2004; 291 (12): 1464-1470

3 Barsky AJ, Goodson JD, Lane RS, Cleary PD. Psychosom Med 1988; 50 (5): 510-519

4 Bass C, Murphy M. J Psychosom Res 1995; 39 (4): $403-$ 427

5 Bhui K, Bhugra D. Int J Soc Psychiatry 2001; 47 (3): 1-9

6 Brown FW, Golding JM, Smith GR Jr. Psychosom Med 1990; 52 (4): 445-451

7 Castillo R, Waitzkin H, Ramirez Y, Escobar Jl. Arch Fam Med 1995; 4 (7): 637-646

8 Dickinson WP, Dickinson LM, deGruy FV et al. Gen Hosp Psychiatry 2003; 25 (1): 1-7

9 Dickinson WP, Dickinson LM, deGruy FV et al. Ann Fam Med 2003; 1 (4): 228-235

10 Fava GA, Mangelli L. Hypochondriasis and anxiety disorders. In: Starcevic V, Lipsitt DR (eds). Hypochondriasis: Modern Perspectives on an Ancient Malady. Oxford: Oxford University Press, 2001: 89-102

11 Fink P, Sørensen L, Engberg $M$ et al. Psychosomatics 1999; 40 (4): 330-338

12 Gül V, Öner E, Uyar K. Psychoneuro 2008; 34 (2): 97-99

13 Gureje O, Simon GE, Ustun TB, Goldberg DP. Am J Psychiatry 1997; 154 (7): 989-995

14 Jackson JL, O’Malley PG, Kroenke K. CNS Spectr 2006; 11 (3): 212-222

15 Jackson JL, Passamonti M. J Gen Intern Med 2005; 20 (11): 1032-1037

16 Keeley R, Smith M, Miller J. Arch Fam Med 2000; 9 (1): 46-54

17 Kirmayer LJ, Young A. Psychosom Med 1998; 60 (4): 420-430

18 Kisely S, Goldberg D, Simon G. Psychol Med 1997; 27 (5): 1011-1019

19 Köster I, Celayir-Erdogan N. Gesundheitswesen 2003; 65 (5): 304-311
Somatisation disorder and psychic co-morbidity - Diagnosis and therapeutic options with Turkish Patients in Germany as examples

Definite progress has already been achieved in diagnosis and therapy of somatisation disorder. However, a new dimension has been introduced to the pathophysiology of this disease pattern by ethnic and cultural aspects. An important part is especially played by co-morbidity in respect of pathology, clinical presentation, treatment and prognosis. It is suspected that a therapy-resistant somatisation disorder is as a matter of fact at least due to psychiatric co-morbidity. In case of co-morbidity it is recommended to treat the co-morbidity first to enable recovery or alleviation of the somatisation disorder. Although many papers on somatisation and ethnicity have already been published, there is unfortunately a scarcity of papers on somatisation disorder and co-morbidity, particularly on psychiatric co-morbidity in migraine, especially in Turkish patients. It is the purpose of the present study to examine psychiatric co-morbidity and to draw special attention to the social and cultural aspects in Turkish patients in Germany who are suffering from a somatisation disorder.

Key words

Somatisation disorder - co-morbidity - culture and ethnicity - Turkish patients

20 Kroenke K, Spitzer RL, Williams JB et al. Arch Fam Med 1994; 3 (9): 774-779

21 Kroenke K, Swindle R. Psychother Psychosom 2000; 69 (4): 205-215

22 Machleidt W, Calliess IT. Die Psychiatrie 2005; 2 (2): 77-84

23 Machleidt W. Psychiat Prax 2005; 32: 55-57

24 Noyes R, Reich J, Clancy J, O'Gorman TW. BrJPsychiatry 1986; 149: 631-635

25 O’Malley PG, Jackson JL, Santoro J et al. J Fam Pract 1999; 48 (12): 980-990

26 Pette M, Pachaly J, David M. Ethn Health 2004; 9 (2): 213-223

27 Piccinelli M, Rucci P, Ustün B, Simon G. Psychol Med 1999; 29 (3): 677-688

28 Rost KM, Akins RN, Brown FW, Smith GR. Gen Hosp Psychiatry 1992; 14 (5): 322-326

29 Russo J, Katon W, Sullivan M, Clark M, Buchwald D. Psychosomatics 1994; 35 (6): 546-556

30 Sauer N, Eich W. Dtsch Ärztbl 2007; 104 (1-2): A 4553

31 Simon GE, Gureje O. Arch Gen Psychiatry 1999; 56 (1): 90-95

32 Simon GE, VonKorff M. Am J Psychiatry 1991; 148 (11): 1494-1500

33 Smith RC, Gardiner JC, Armatti S et al. Med Care 2001; 39 (9): 968-978

34 Speckens AE, Van Hemert AM, Bolk JH, Rooijmans HG, Hengeveld MW. Psychol Med 1996; 26 (4): 745-752

35 Ulusahin A, Basoglu M, Paykel ES. Soc Psychiatry Psychiatr Epidemiol 1994; 29 (1): 31-39

36 van den Brink Y. J Transcult Nurs 2003; 14(2): 146-154

37 van Hemert AM, Hengeveld MW, Bolk JH et al. Psychol Med 1993; 23 (1): 167-173

38 Waitzkin H, Magaña H. Soc Sci Med 1997; 45 (6): $811-$ 825

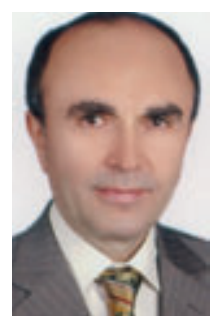

\section{Korrespondenz}

Dr./Univ. Bursa Vahdet Gül

Praxis für Psychiatrie

Königsberger Straße 3

75417 Mühlacker

praxisdrgul@aol.com 